\title{
Simultaneous Bilateral Cochlear Implantation in Posttraumatic Temporal Bone Fracture under Local Anaesthesia - Case Report and Literature Review
}

\author{
Lucas Resende Lucinda Mangia, MD*, Fellipy Martins Raymundo, MD, Nicole Tássia \\ Amadeu, MD and Rogério Hamerschmidt, PhD
}

Department of Otolaryngology and Head and Neck Surgery, Complexo Hospital de Clínicas - Universidade Federal do Paraná (CHC-UFPR), Curitiba, Brazil

\begin{abstract}
Bilateral deafness caused by temporal bone fracture is an uncommon condition. Precocious simultaneous bilateral cochlear implantation $(\mathrm{SiCl})$ is a valuable option for experienced surgical teams, which can prevent unfavorable results due to cochlear remodeling. In this paper we describe the first successful case of $\mathrm{SiCl}$ with local anaesthesia performed on an adult patient who suffered severe head trauma.
\end{abstract}

Keywords

Temporal bone fracture, Cochlear implantation, Local anaesthesia, Simultaneous bilateral cochlear implantation, Head trauma

\section{Introduction}

Temporal bone fracture is a well-known cause of sensorineural hearing loss (SNHL) [1]. Severe head injury can cause bilateral deafness in rare cases, and might require cochlear implantation $(\mathrm{Cl})$ for effective rehabilitation. Injuries to the otic capsule, either by the fracture line itself, cochlear concussion or damage to the cochlear branch of the vestibulocochlear nerve are potential limitators [2].

Simultaneous bilateral cochlear implantation ( $\mathrm{SiCl}$ ) in adults with acquired hearing loss might anticipate the benefits of binaural hearing and potentially reduce overall surgical costs, decrease absenteeism rates and diminish anesthetic and hospital risks. There is a concern about $\mathrm{Cl}$ after temporal bone trauma, which might lead to secondary fibrosis of the cochlear lumen or labyrinthitis ossificans and hinder the insertion and progression of the electrodes array into the cochlea [3]. Thus, precocious $\mathrm{SiCl}$ in this context might have an additional advantage of preventing unfavorable results due to cochlear remodeling.

Most services in the world prefer general anaesthesia to perform cochlear implantation. We herein describe the first bilateral cochlear implantation carried out under local anaesthesia following deafness secondary to a bilateral transversal temporal bone fracture. A literature review is also included.

\section{Case Report}

A 40-year-old male presented with bilateral deafness fol- lowing a fall-related head trauma two months before consultation. He had no history of facial paralysis, vertigo or neurological deficits. The otoscopic examination was normal. No clinical evidences of peripheral facial nerve palsy were found (House-Brackmann grade 1). Brain computed tomography (CT) scan was performed. It showed no signs of intracranial injury, however the images revealed bilateral complex transversal temporal fractures. Fracture lines affected both otic capsules (Figure 1A, Figure 1B and Figure 1C) and ran closely to the fundus of the internal auditory meatus (Figure 1D).

A pure-tone audiometry revealed right-sided anacusia and profound hearing loss on the left ear, with minimal residual hearing. Speech audiometry showed no mono- or disyllabic recognition ability (word recognition score $=0 \%$ ). A type

*Corresponding author: Lucas Resende Lucinda Mangia, Department of Otolaryngology and Head and Neck Surgery, Hospital de Clínicas Complex - Federal University of Paraná, R Gen Carneiro, 181 - Alto da Glória, Curitiba, Paraná, 80060-900, Brazil, Tel: +55-41-992855494

Accepted: March 13, 2021

Published online: March 15, 2021

Citation: Mangia LRL, Raymundo FM, Amadeu NT, et al. (2021) Simultaneous Bilateral Cochlear Implantation in Posttraumatic Temporal Bone Fracture under Local Anaesthesia - Case Report and Literature Review. J Head Neck Surg 3(1):137-140 


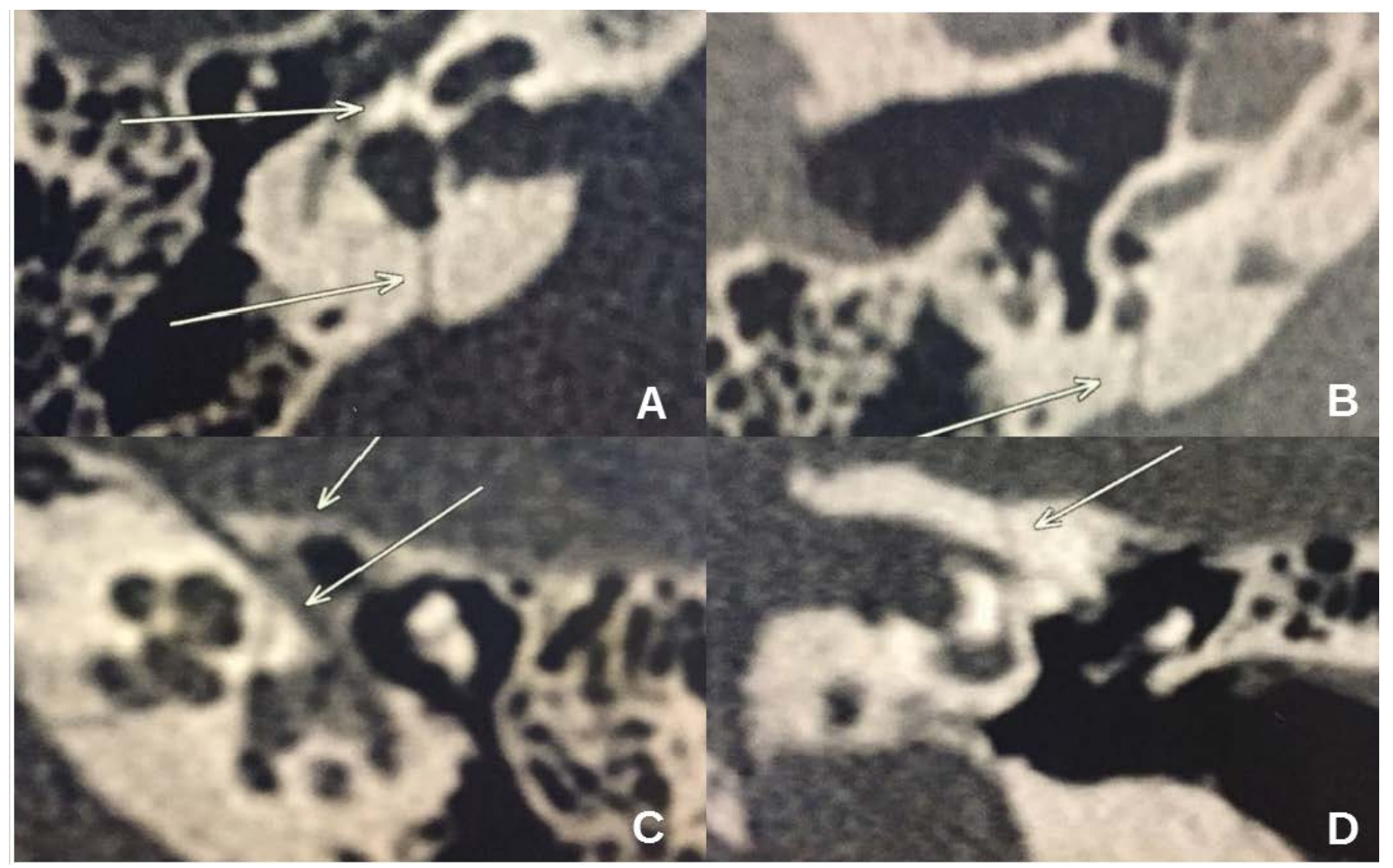

Figure 1: Temporal bone CT-scan showing a complex transversal bilateral temporal bone fracture. White arrows indicate fracture lines. (A) Fracture lines running across the vestibule and near the cochlea on the right side, axial view; (B) An inferior fracture line reaches the round window on the right side, axial view; (C) Transversal fracture lines within the otic capsule next the cochlea on the left side, axial view; (D) A superior transversal fracture line runs towards the fundus of the internal auditory meatus.

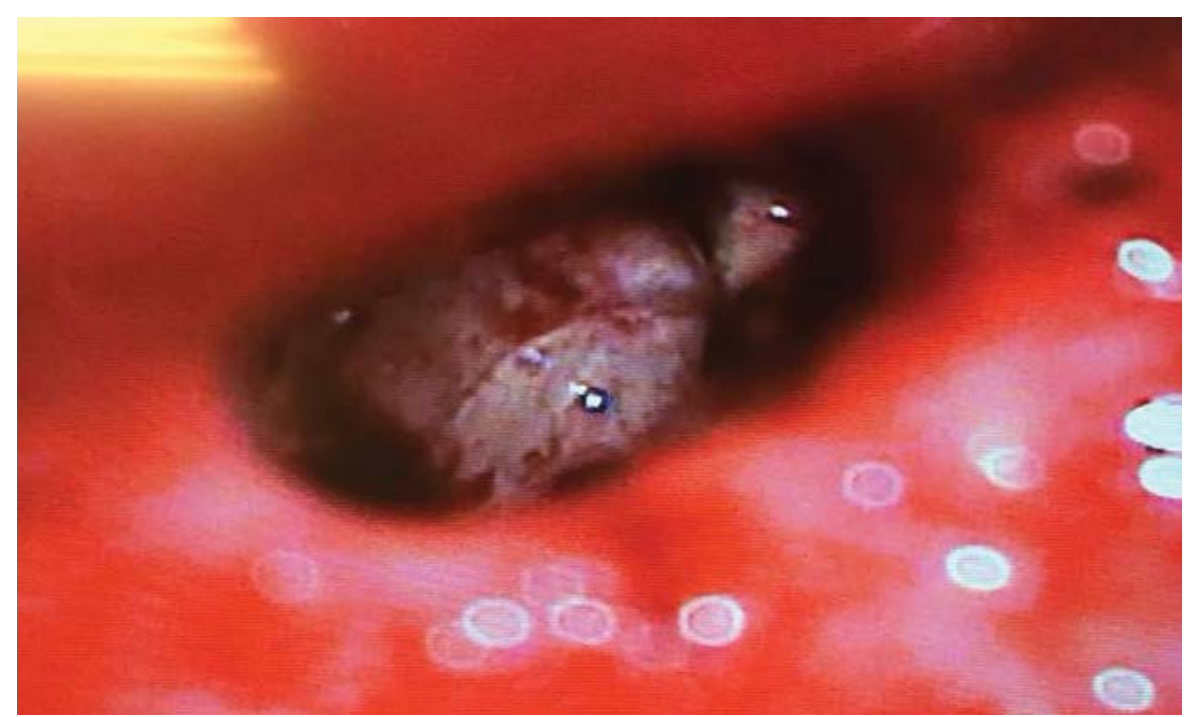

Figure 2: Intraoperative microscopic view across the posterior timpanotomy showing a fracture line affecting the cochlea very close the round window.

A tympanogram was observed bilaterally. Brainstem evoked response audiometry (BERA) failed to identify any electrophysiological response at the maximum stimulation intensity provided by the device ( $99 \mathrm{DBHL}$ ) in both sides.

The patient had no benefit with hearing aids, with thresholds on open-field audiometry greater than $90 \mathrm{~dB}$. Besides, speech comprehension tests were unfavorable. The Ling Six-Sound Test, the monosyllabic and dissyllabic word perception index and the sentence recognition performance in both closed and open-set response formats did not show any speech perception ability. Audio-visual speech recognition rates reached $20 \%$. 
Citation: Mangia LRL, Raymundo FM, Amadeu NT, et al. (2021) Simultaneous Bilateral Cochlear Implantation in Posttraumatic Temporal Bone Fracture under Local Anaesthesia - Case Report and Literature Review. J Head Neck Surg 3(1):137-140

Surgery for bilateral simultaneous cochlear implantation was indicated and performed under local anesthesia and sedation, after patient's consent. During the procedure, it was possible to identify a fracture line in the basal turn of the cochlea bilaterally (Figure 2 ), near the round window niche. Nonetheless, the complete insertion of the electrode array was possible with no need of cochleostomies. There were no anesthetic intercurrences. The patient did not report any discomfort in the postoperatively, and was discharged home with standard instructions and follow up.

After 30 days, he returned for implant activation and programming. At this point, he could immediately detect sounds and even showed signs of improved voice perception. A postoperative CT scan showed correct placement of the electrodes array within the cochlea (Figure 3).

\section{Discussion}

Head trauma is a frequent condition and secondary hearing loss is a possible well-established sequela. The prevalence of temporal bone involvement among skull fractures ranges from 18 to $22 \%[1,4]$. Signs and symptoms of temporal bone fractures include hemorrhagic otorrhea, hemotympanum, tympanic membrane perforation, vertigo, hearing loss, facial paralysis and nystagmus [1].

Temporal bone fractures are traditionally classified according to the direction of the fracture line when compared to the long axis of the petrous pyramid [5]. Longitudinal fractures are the most common, corresponding to $80-90 \%$ of total cases. It is usually caused by an impact to the temporoparietal region and is often associated with damages to the ossicular chain and tympanic membrane. On the other hand, transversal fractures occur due to front-occipital traumas and is associated with increased risk of facial nerve injury $[1,6]$.

A novel classification system, proposed by Kelly and Tami [7], based on the existence of injuries fracturing the otic capsule, seems to be more adequate for correlating the fracture type with clinical outcomes [8]. It is known that most patients (94-97\%) have fractures sparing the otic capsule, whose audiograms usually describe a conductive hearing loss corre- sponding to damage to the tympano-ossicular system. Conversely, those with violation of the otic capsule (3-6\%) have noticeable higher prevalence of facial nerve paralysis (30$50 \%)$, SNHL and perilymphatic fistula [7].

Hearing loss following temporal bone fracture might occur in $24-81 \%$ of patients due to involvement of different parts of the auditory system, from the outer ear canal to the auditory cortex. The most common presentation is a conductive impairment secondary to hemotympanum or tympanic membrane perforation, from which the patients usually present full recovery. However, if persistent, it might indicate damage to the ossicular chain or even a secondary cholesteatoma [9].

SNHL, isolated or of mixed-type, accounts for $20 \%$ of the cases [9] and can be explained by an abnormal movement of the stapes into the oval window, resulting in increased pressure within the membranous labyrinth and damage to the organ of Corti; travelling pressure waves directly affecting the cochlea; a traumatic perilymphatic fistula; impaired blood supply to the inner ear as result of phenomena such as vascular thrombosis or increased intracranial pressure; or direct injury of the cochlear nerve or central auditory pathways [2]. Nevertheless, labyrinthine concussion might generate microcirculatory disturbances, hypoxia and consequent unbalance of the endo/perilymphatic hemostasis, and patients may experience severe or profound hearing loss despite no macroscopic alterations. Occasionally, a concomitant acoustic trauma can lead to hearing impairment [9].

Cochlear implants are frequently used for rehabilitation of bilateral severe to profound SNHL. Despite the well-known advantages obtained with the unilateral procedure, it does not restore binaural hearing. Bilateral $\mathrm{Cl}$ significantly improve sound localization and perception in noisy environments. These benefits might have a positive impact in terms of social integration, emotional well-being and cognition. Moreover, the bilateral procedure seems to enhance work performance and diminishes work-related stress and fatigue [10].

There are only few studies on the results of $\mathrm{Cls}$ among patients with hearing loss due to trauma. Alves, et al. [4] found that results obtained by post lingual implanted adults who

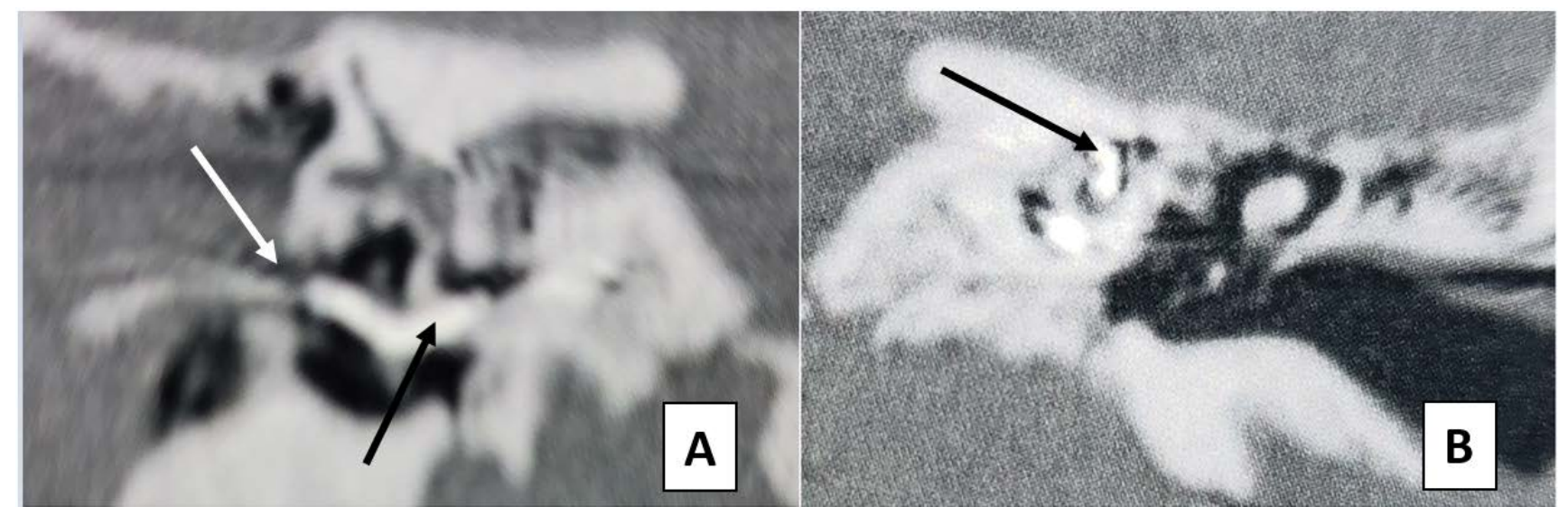

Figure 3: Postoperative computed tomography images showing the proper insertion of the electrodes array. (A) Electrodes array running across the posterior tympanotomy (white arrow) and into the cochlea through the round window (black arrow), coronal view, right side; (B) Electrodes within the cochlea (black arrow), axial view, left side. 
Citation: Mangia LRL, Raymundo FM, Amadeu NT, et al. (2021) Simultaneous Bilateral Cochlear Implantation in Posttraumatic Temporal Bone Fracture under Local Anaesthesia - Case Report and Literature Review. J Head Neck Surg 3(1):137-140

had suffered head trauma were globally poorer. Concomitant brain lesions that might impair sound perception could help explain such a worse performance. Besides, phenomena such as labyrinthitis ossificans, fibrosis and vicious ossification might occur after cochlear trauma and hinder the insertion of the electrodes array or their perfect functioning after insertion [3]. Thus, precocious implantation in this setting is preferable and, if the bilateral procedure is recommended, simultaneous approach seems more advantageous.

Most services prefer to perform $\mathrm{Cl}$ under general anesthesia. Nonetheless, otologic surgeries have been successfully performed under local anesthesia and sedation and are associated with lower morbidity and reduced costs [10-13]. In addition, performing surgery without general anesthesia might be a viable alternative for patients with increased anesthetic risk. However, it demands an experienced and welltrained surgical team [10-13]. Preoperative counseling plays a major role in predicting overall patient cooperation during the procedure. The possibility of intraoperative vestibular stimulation needs to be informed to the patient, in order to avoid unexpected discomfort. Due to the patient's hearing impairment, gestural and sign language are the only possible methods of communication during surgery [13].

Vaid, et al. [14] reported one case of a patient who underwent a successful $\mathrm{SiCl}$ under local anesthesia. This patient presented vestibular symptoms during the electrodes insertion, which ameliorated with administration of intramuscular prochlorperazine mesylate. The authors emphasized that intraoperative telemetry allowed the patient to detect sounds soon after the procedure.

\section{Conclusion}

We described an unusual case of an adult patient with bilateral profound hearing loss secondary to head trauma, which was successfully submitted to $\mathrm{SiCl}$ under local anesthesia. The procedure provided satisfactory outcomes, both in terms of safety and efficacy. Patients who had suffered head injury with temporal bone fracture are suitable for $\mathrm{Cl}$, as long as the standard criteria are met. This includes bilateral implantation whenever possible and necessary. Local anesthesia with sedation is feasible for cochlear implants in cooperative adults, even when the procedure is bilateral.

\section{Funding}

This research did not receive any specific grant from funding agencies in the public, commercial, or not-for-profit sectors.

\section{Declarations of Interest}

The authors declare no conflicts of interest.

\section{References}

1. Juliano AF, Ginat DT, Moonis G (2015) Imaging review of the temporal bone: Part B, traumatic, postoperative, and noninflammatory nonneoplastic conditions. Radiology 276: 655-672.

2. Bergemalm P (2003) Progressive hearing loss after closed head injury : A predictable outcome ? Acta Otolaryngol 123: 836-845.

3. Lee J, Nadol JBJ, Eddington DK (2011) Factors associated with incomplete insertion of electrodes in cochlear implant surgery: A histopathologic study. Audiol Neurootol 16: 69-81.

4. Alves M, Martins JH, Moura JE, et al. (2014) Auditory rehabilitation after cochlear implantation in adults with hearing impairment after head trauma. Cochlear Implants Int 15: 312-317.

5. Johnson F, Semaan MT, Megerian CA (2008) Temporal bone fracture : Evaluation and management in the modern era. Otolaryngologic Clinics 41: 597-618.

6. Ghorayeb BY, Yeakley JW (1992) Temporal bone fractures: Longitudinal or oblique? The case for oblique temporal bone fractures. Laryngoscope 102: 129-134.

7. Kelly K, Tami T (1994) Temporal bone and skull base trauma. Jackler RK, Brac. Neurotol 1127-1147.

8. Brodie H, Thompson T (1997) Management of complications from 820 temporal bone fractures. Am J Otol 18: 188-197.

9. Zimmer A, Reith W (2014) Traumata des Felsenbeins. Radiologe 54: 340-345.

10. Härkönen K, Kivekäs I, Rautiainen M, et al. (2015) Sequential bilateral cochlear implantation improves working performance , quality of life, and quality of hearing. Acta Otolaryngol 135: 440-446.

11. Hamerschmidt R, Mocellin M, Gasperin AC, et al. (2010) Local anesthesia for cochlear implant surgery: A possible alternative. Braz J Otorhinolaryngol 76: 561-564.

12. Hamerschmidt R, Mocellin M, Gasperin A, et al. (2011) Local anesthesia for cochlear implant surgery: Low risks for the patient and few costs for the hospital, a rational alternative. Rev Laryngol Otol Rhinol (Bord) 132: 227-229.

13. Hamerschmidt R, Moreira ATR, Wiemes GRM, et al. (2013) Cochlear implant surgery with local anesthesia and sedation: comparison with general anesthesia. Otol Neurotol 34: 75-78.

14. Vaid N, Jana JJ, Kothadiya A, et al. (2016) Bilateral cochlear implantation under local anaesthesia in a young adult - A case report. Cochlear Implants Int 17: 207-210.

DOI: $10.36959 / 605 / 556$

Copyright: (c) 2021 Mangia LRL, et al. This is an open-access article distributed under the terms of the Creative Commons Attribution License, which permits unrestricted use, distribution, and reproduction in any medium, provided the original author and source are credited. 